\title{
THE RESOLVED SHEAR STRESS ON THE NON-ACTIVE SLIP SYSTEMS IN TAYLOR/BISHOP-HILL MODELS FOR FCC POLYCRYSTALS
}

\author{
B. Clausen*1, T. Leffers*, T. Lorentzen*, O.B. Pedersen* \\ *Materials Research Department, Risø National Laboratory, DK-4000 Roskilde, Denmark \\ P. Van Houtte** \\ **Department MTM, Katholieke Universiteit Leuven, B-3001 Heverlee, Belgium
}

(Received May 18, 1999)

(Accepted August 31, 1999)

Keywords: Theory and modelling; Mechanical properties; Constitutive equations; Crystal structure; Resolved shear stress

\section{$\underline{\text { Introduction }}$}

According to the Bishop-Hill rate-insensitive model (1-3) for the plastic deformation of polycrystals (in casu fcc polycrystals) there are, in a grain of a given orientation, 6 or 8 potentially active $\{111\}\langle 110\rangle$ slip systems with a resolved shear stress equal to the critical resolved shear stress. Out of these, at least 5 are active according to Taylor (4) (in reality the Taylor model is identical to the Bishop-Hill model with some additional rule to select the active slip systems (5) — to solve the "Taylor ambiguity"). For the 6 or 4 slip systems which are not potentially active Bishop and Hill only state that the resolved shear stress is lower than the critical resolved shear stress. In the present work we shall show that it is actually zero, and we shall discuss certain implications.

\section{Model Observations}

The original observations of the zero value of the resolved shear stress on the non potentially active slip systems in fcc polycrystals were made for self-consistent models of the Kröner (6) and Hutchinson (7) type (the Kröner-type model is described in (8) and the Hutchinson-type model is described in (9)). In Kröner's model all grains reach vertex stresses on the yield surface, in Hutchinson's model only relatively few grains reach vertex stresses. In both models the computer printed out the 12 resolved shear stresses in each grain, and it turned out that whenever vertex stresses were reached, there were 6 or 8 slip systems with resolved shear stresses practically equal to the critical shear stress and 6 or 4 slip systems with resolved shear stress practically equal to zero. When practically must be added, it is due to the structures of the two computer programs (not to be described here) which lead to finite deviations from the exact vertex stresses.

Inspired by these "empirical" observations we provide, in the next section, a strict mathematical proof of the zero value of the resolved shear stresses on the slip system which are not potentially active.

\footnotetext{
${ }^{1}$ Present address: Los Alamos National Laboratory, Mail Stop H805, Los Alamos, NM 87545, USA. 
TABLE 1

The Parameters $S_{i j}$ Corresponding to the Five Types of Vertices Numbered (i) to (v) by Bishop and Hill (2)

\begin{tabular}{lclrlll}
\hline Type & $S_{11}$ & $\mathrm{~S}_{22}$ & $\mathrm{~S}_{33}$ & $\mathrm{~S}_{23}$ & $\mathrm{~S}_{31}$ & $\mathrm{~S}_{12}$ \\
\hline i & 1 & 1 & -2 & 0 & 0 & 0 \\
ii & 0 & 0 & 0 & 3 & 0 & 0 \\
iii & -1.5 & 1.5 & 0 & 0 & 0 & 1.5 \\
iv & 0.5 & 0.5 & -1 & 1.5 & 1.5 & 0 \\
v & 0 & 0 & 0 & 1.5 & 1.5 & 1.5 \\
\hline
\end{tabular}

See eq. (1) for the value of the deviatoric stresses.

\section{Analysis}

Bishop and Hill $(1,2)$ have constructed the yield locus of an fcc single crystal. In the five-dimensional deviatoric stress space (a $\sigma_{11}+\sigma_{22}+\sigma_{33}=0$ section of the six-dimensional stress space) it looks like a polyhedron with many facets. It has 56 vertices. The corresponding deviatoric stresses are called vertex stresses. Hill's Maximum Work Principle for rate-insensitive plasticity was then used to demonstrate that the stress which corresponds to a prescribed plastic strain vector will nearly always be a vertex stress, except when the prescribed plastic strain happens to be exactly orthogonal to one of the facets or ribs of the polyhedron-shaped yield locus. The probability that this happens is infinitesimal, unless a special combination of a crystal orientation and a strain vector is chosen on purpose.

Bishop and Hill (2) arrange the 56 vertices of an fcc crystal in 5 types of vertex stresses. Table 1 is derived from their data. It shows the deviatoric stress of one of the vertices for each type. The other vertices can be derived from these by applying the symmetry operators of the crystal.

The table actually gives the parameters $S_{i j}$, from which the deviatoric stresses are derived as follows ( $\tau^{c}$ is the critical resolved shear stress):

$$
\sigma_{i j}^{\prime}=\frac{\sqrt{6}}{2} S_{i j} \tau^{c}
$$

In what follows, each of these stresses will be applied to the crystal in order to calculate the resolved shear stress on all slip systems. The resolved shear stress on slip system $s$ is given by:

$$
\tau_{s}^{r}=\sigma_{i j}^{\prime} M_{i j}^{s}
$$

$M_{i j}^{s}$ is a geometrical constant which is defined as follows $(1,2)$ :

$$
M_{i j}^{s}=\frac{1}{2}\left(n_{i} b_{j}+n_{j} b_{i}\right)
$$

in which $\mathbf{n}$ is the unit vector normal to the slip plane with Miller indices $\left(h_{1} h_{2} h_{3}\right)$. For fcc materials these are $\{111\}$-planes, and we have:

$$
n_{i}=\frac{1}{\sqrt{3}} h_{i}
$$

b is the unit vector in the slip direction with Miller indices $\left[u_{1} u_{2} u_{3}\right]$. For fcc materials these are $\langle 110\rangle$-directions, and we have:

$$
b_{i}=\frac{1}{\sqrt{2}} u_{i}
$$


TABLE 2

$N_{i j}=h_{i} u_{j}+h_{j} u_{i}$ Parameters for All Slip Systems (see also Eq. (6))

\begin{tabular}{|c|c|c|c|c|c|c|c|c|c|c|c|c|}
\hline$s$ & $h_{1}$ & $h_{2}$ & $h_{3}$ & $u_{1}$ & $u_{2}$ & $u_{3}$ & $N_{11}$ & $N_{22}$ & $N_{33}$ & $N_{23}$ & $N_{31}$ & $N_{12}$ \\
\hline 1 & 1 & 1 & 1 & 0 & 1 & -1 & 0 & 2 & -2 & 0 & -1 & 1 \\
\hline 2 & 1 & 1 & 1 & -1 & 0 & 1 & -2 & 0 & 2 & 1 & 0 & -1 \\
\hline 3 & 1 & 1 & 1 & 1 & -1 & 0 & 2 & -2 & 0 & -1 & 1 & 0 \\
\hline 4 & -1 & 1 & 1 & 0 & 1 & -1 & 0 & 2 & -2 & 0 & 1 & -1 \\
\hline 5 & -1 & 1 & 1 & 1 & 0 & 1 & -2 & 0 & 2 & 1 & 0 & 1 \\
\hline 6 & -1 & 1 & 1 & 1 & 1 & 0 & -2 & 2 & 0 & 1 & 1 & 0 \\
\hline 7 & 1 & -1 & 1 & 0 & 1 & 1 & 0 & -2 & 2 & 0 & 1 & 1 \\
\hline 8 & 1 & -1 & 1 & -1 & 0 & 1 & -2 & 0 & 2 & -1 & 0 & 1 \\
\hline 9 & 1 & -1 & 1 & 1 & 1 & 0 & 2 & -2 & 0 & 1 & 1 & 0 \\
\hline 10 & 1 & 1 & -1 & 0 & 1 & 1 & 0 & 2 & -2 & 0 & 1 & 1 \\
\hline 11 & 1 & 1 & -1 & 1 & 0 & 1 & 2 & 0 & -2 & 1 & 0 & 1 \\
\hline 12 & 1 & 1 & -1 & 1 & -1 & 0 & 2 & -2 & 0 & 1 & -1 & 0 \\
\hline
\end{tabular}

Eqs. (3-5) combined result in:

$$
M_{i j}^{s}=\frac{1}{2 \sqrt{6}}\left(h_{i} u_{j}+h_{j} u_{i}\right)=\frac{1}{2 \sqrt{6}} N_{i j}^{s}
$$

Note that the parameters $N_{i j}^{s}$ are integer numbers which can easily be calculated by hand. They are given by Table 2 (the superscript $S$ is omitted in the table).

The resolved shear stresses $\tau_{s}^{r}$ can now be calculated on all slip systems for the five types of vertices. After some elaboration, it follows from eqs. (1-6) that

$$
\tau_{s}^{r}=\frac{1}{6} Q_{s} \tau^{c}
$$

with

$$
Q_{s}=S_{i j} N_{i j}^{s}
$$

TABLE 3

$Q_{s}$-Values (see Eqs. 7-8) for Each of the 5 Types of Vertices, and Each of the 12 Slip Systems

\begin{tabular}{lrrrrr}
\hline$s$ & (i) & (ii) & (iii) & (iv) & (v) \\
\hline 1 & 6 & 0 & 6 & 0 & 0 \\
2 & -6 & 6 & 0 & 0 & 0 \\
3 & 0 & -6 & -6 & 0 & 0 \\
4 & 6 & 0 & 0 & 6 & 6 \\
5 & -6 & 6 & 6 & 0 & 6 \\
6 & 0 & 6 & 6 & 6 & 6 \\
7 & -6 & 0 & 6 & 0 & 6 \\
8 & -6 & 6 & -6 & 6 & 6 \\
9 & 0 & 0 & 6 & 6 & 6 \\
10 & 6 & 6 & -6 & 6 & 0 \\
11 & 6 & 6 & & 0 & 0 \\
12 & 0 & 0 & & & 6 \\
\hline
\end{tabular}

These values are proportional to the resolved shear stresses. 
$S_{i j}$ is given by Table 1 , and $N_{i j}^{s}$ can be found in Table 2. Eq. (8) then leads to Table 3 (these calculations can also easily be made by hand).

Inspection of Table 3 leads to the following conclusions:

- The only values present are 0,6 or -6 . In view of eq. (7) this means that the resolved shear stresses on the slip systems can only take the values $0, \tau^{c}$ or $-\tau^{c}$. In other words, the vertex stresses will either induce enough shear stress to activate a slip system or no shear stress at all. Note that this conclusion is valid for fcc materials with $\{111\}\langle 110\rangle$ slip, but not necessarily for other sets of slip systems.

-The vertex types (i), (ii), (iii) may activate 8 slip systems, whereas the types (iv) and (v) only activate 6 slip systems.

The property which has been discovered here also has a geometrical meaning in stress space. Each facet of a single crystal yield locus corresponds to a slip system. It is part of a hyperplane which is described by the following linear equation (note: in the following equations, ordinary stress components as well as deviatoric stress components can be used):

$$
\sigma_{i j} M_{i j}^{s}=+\tau^{c} \text { or }-\tau^{c}
$$

The coefficients $M_{i j}^{s}$ can be seen as the components of a vector which is normal to the hyperplane. Conversely, each slip system corresponds to two facets which are parallel to each other, one for slip in the positive sense, and one for slip in the negative sense.

The following equation holds for a vertex stress $\sigma^{V}$ which would induce a zero resolved shear stress on this slip system (see eq. (2)):

$$
\sigma_{i j}^{V} M_{i j}^{s}=0
$$

Geometrically, this means that the vector $\sigma^{V}$ and the vector $M_{i j}^{s}$ are orthogonal to each other; in other words, the vertex stress is parallel to the hyperplanes described by eq. (9), i.e. to the facets which correspond to the slip system $s$.

It can be concluded from this that for fcc materials only two types of relationship are possible between a vector representing a vertex stress $\sigma^{V}$ and the two facets which correspond to a particular slip system. These two types are:

(1) the end point of the vector representing $\sigma^{V}$ is on one of the two facets; the vertex stress may activate the slip system;

(2) the vector $\sigma^{V}$ is parallel to the facets.

This property can be seen in any yield locus section of an fcc crystal, such as Fig. 1. The vertices seen in such a section are the projections of genuine deviatoric vertices on the section plane. The projection direction is the direction of the hydrostatic stress.

\section{$\underline{\text { Discussion }}$}

It should be underlined that the zero value of the resolved shear stress on the non-potentially active slip systems is unique for fcc materials with their $12\{111\}\langle 110\rangle$ slip systems (and for bcc materials with $\{110\}\langle 111\rangle$ slip only).

One may view the zero values as a curiosity. Even so, it is a curiosity which came to us as a great surprise. However, we think there are important implications for the ambiguity problem in the 


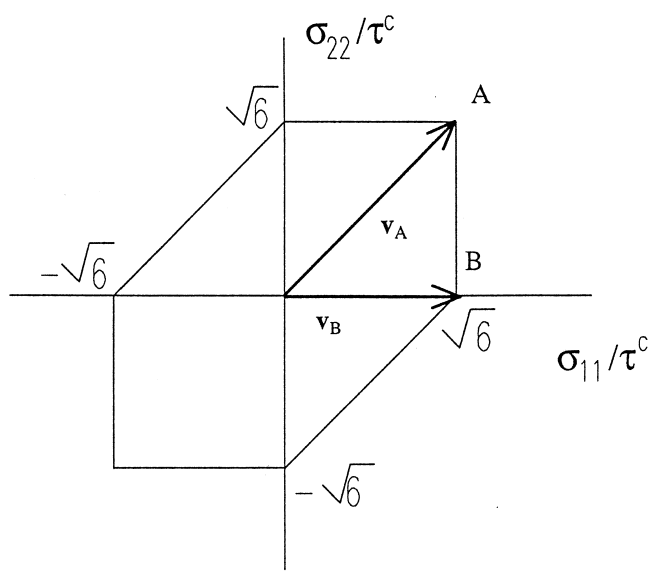

Figure 1. Yield locus section of an fcc single crystal. The vectors $\mathbf{v}_{\mathrm{A}}$ and $\mathbf{v}_{\mathrm{B}}$ representing the vertex stresses A and B are parallel to the facets which correspond to non-activated slip systems.

Taylor/Bishop-Hill model complex (without rate sensitivity) and for the suggestion that the ambiguity problem may be solved once and forever by the introduction of rate sensitivity (10).

In a panel discussion at ICOTOM 8 (11) two different interpretations of the ambiguity problem were presented: (i) that the ambiguity, as it appears in the rate-insensitive Taylor/Bishop-Hill models, reflects a physical reality, viz. that there are a number of equally plausible solutions for the slip pattern in polycrystals, and (ii) that the ambiguity problem is a pseudo problem associated with the unrealistic assumption of strict rate insensitivity which is eliminated by even the slightest rate sensitivity ("rate sensitivity at the limit of rate insensitivity").

In our opinion the zero value of the resolved shear stresses on the non potentially active slip systems in the rate-insensitive approach is a strong support for interpretation (i) — or rather a strong argument against interpretation (ii). We are obviously, even with a certain rate sensitivity, very close to a situation with zero resolved shear stress on 6 or 4 slip systems and hence with practically no slip on these systems. Thus, the apparent solution to the ambiguity problem by a slight rate sensitivity relies on marginal differences in the resolved shear stresses on the same 6 or 8 slip systems which may be active in the rate-insensitive approach. These marginal differences are determined by the detailed structure of the computer programs used rather than by real physics. One of the "non-physical" components of these computer programs is the exact form of the relation between resolved shear stress and slip rate for the individual slip systems. Such marginal differences do not a priori provide a slip pattern which is "better" than that provided by an appropriate additional rule in the Taylor/Bishop-Hill model.

Thus, in the universe of polycrystal models with many active slip systems-whether they are rate-sensitive or rate-insensitive-we must, at least for fcc materials, accept that the overall model per se does not provide an unambiguous selection of active slip systems. In rate-insensitive models additional rules derived from physical ideas about the interaction of the different slip systems (e.g. (10)), must be included. In rate-sensitive models the additional rules are hidden in the structure of the computer program. We do not claim that rate-insensitive models are better than rate-sensitive models. On the contrary we accept that rate-sensitive models may be more efficient. We only claim that rate-sensitive models for fcc materials do not provide solutions which are, in the real sense of the word, more unique than those provided by rate-insensitive models. 


\section{Summary}

It is demonstrated for fcc materials with $\{111\}\langle 110\rangle$ slip that the resolved shear stress on the slip systems which are not potentially active according to the rate-insensitive Taylor/Bishop-Hill model is zero. It is argued that this implies an effective ambiguity in the selection of slip systems even with rate sensitivity.

\section{Acknowledgement}

The work at Ris $\varnothing$ was carried out within the Engineering Science Centre for Structural Characterization and Modelling of Materials.

\section{$\underline{\text { References }}$}

1. J.F.W. Bishop, and R. Hill, Phil. Mag. 42, 414 (1951).

2. J.F.W Bishop, and R. Hill, Phil. Mag. 42, 1298 (1951).

3. J.F.W Bishop, Phil. Mag. 44, 5 (1953).

4. G.I. Taylor, J. Inst. Metals 62, 307 (1938).

5. L. Dezillie, P. Van Houtte, and E. Aernoudt, in Proceedings ICOTOM 8, ed. J.S. Kallend and G. Gottstein, p. 357, The Metallurgical Society, Warrendale, PA (1988).

6. E. Kröner, Acta Metall. 9, 155 (1961).

7. J.W. Hutchinson, Proc. R. Soc. London A. 319, 247 (1970).

8. T. Leffers, and O.B. Pedersen, Constitutive Relations and Their Physical Basis, ed. S.I. Andersen et al., p. 401, Ris $\emptyset$ National Laboratory, Roskilde (1987).

9. B. Clausen, T. Lorentzen, and T. Leffers, Acta Mater. 46, 3087 (1998).

10. G.R. Canova, and U.F. Kocks, in Proceedings ICOTOM 7, ed. C.M. Brakman et al., p. 573, The Netherland Society for Materials Science, Zwijndrecht (1984).

11. T. Leffers, R.J. Asaro, J.H. Driver, U.F. Kocks, H. Mecking, C. Tomé, and P. Van Houtte, in Proceedings ICOTOM 8, ed. J.S. Kallend and G. Gottstein, p. 265, The Metallurgical Society, Warrendale, PA (1988). 